\title{
New Primary Ipsilateral Metachronous Breast Tumor: A Case Report
}

\author{
Silvia Cuerda ${ }^{a} \quad$ Natalia Ramírez ${ }^{\mathrm{b}}$ Luis Chara $^{\mathrm{b}}$ \\ Javier Cassinello ${ }^{b}$ \\ ${ }^{a}$ Pharmacy Department and ${ }^{b}$ Medical Oncology Department, Guadalajara \\ University General Hospital, Guadalajara, Spain
}

\section{Key Words}

Breast cancer $\cdot$ Ipsilateral breast tumor relapse $\cdot$ Her-2 $\cdot$ Inflammatory carcinoma $\cdot$ True recurrence $\cdot$ New primary tumor

\begin{abstract}
After breast-conserving treatment, the occurrence of ipsilateral breast tumor relapse raises the concern regarding whether it may represent two distinct types of lesion that it is important to define, a true recurrence (TR) or a new primary tumor (NPT). TR and NPT have different natural histories, prognosis, and in turn different implications for therapeutic management. We report the case of a 35-year-old woman who developed a breast invasive ductal carcinoma, which after receiving breast-conserving treatment with adjuvant chemotherapy, radiotherapy and hormone therapy, developed four years after an inflammatory carcinoma in the same breast, with different expression of immunohistochemical markers than the first breast cancer. The patient was treated with neoadjuvant chemotherapy that allowed the realization of a radical mastectomy with a complete pathological response. We describe the diagnostic and therapeutic approach of ipsilateral breast tumor relapses, along with a review of existing literature.
\end{abstract}

\section{Introduction}

The incidence of ipsilateral breast tumor relapse (IBTR) in patients previously treated for breast carcinoma through breast-conserving surgery, systemic adjuvant therapy and radiation therapy, is estimated to be less than $6 \%$. However, as each case has different clinical relevance (evolution, prognosis and therapeutic management), it is necessary to distinguish in these patients between a true local recurrence (TR) of a previous tumor, and the appearance of a new primary tumor (NPT) [1-4].

Javier Cassinello Espinosa, MD $\mathrm{PhD}$
Servicio de Oncología Médica, Hospital General Universitario de Guadalajara Calle Donante de Sangre $\mathrm{S} / \mathrm{N}$

ES-19002 Guadalajara (Spain)

E-Mail jacaes@sescam.jccm.es 
To do this, we must study the location of the new tumor (distance from the previous tumor), staging (by imaging and biopsy) and determine their histological subtype (through immunohistochemical studies, and the determination of HER-2 and hormone receptor expression).

\section{Case Report}

A 35-year-old premenopausal woman with no relevant medical history of interest, except for a family history of a maternal cousin with breast cancer at 28 years, was referred to the oncologic gynecology department by the palpation of a $1-\mathrm{cm}$ nodule in the right axilla. It was nontender to palpation, but fixed to surface skin structures. Fine needle aspiration cytology was performed, with the pathological result of suspicion of malignancy.

In February 2004, the patient underwent a lumpectomy with axillary lymph node dissection. The pathological diagnosis was moderately differentiated invasive ductal carcinoma (IDC) of the right breast of $0.8 \mathrm{~cm}$ in size, with no metastasis detected in 20 lymph nodes removed $\left(\mathrm{pT}_{1} \mathrm{pN}_{0} \mathrm{M}_{0}\right)$. Immunohistochemical study of the tumor cells showed positive staining for both estrogen receptors (ER) and progesterone receptors (PR) in 50 and 15\%, respectively, and showed negative membrane staining of Her-2 marker. Fluorescence in situ hybridization (FISH) analysis found no HER-2 amplification in the primary tumor. The computer tomography performed after surgical intervention revealed no other organs compromised or distant metastatic extension. Preoperative serum levels of the marker CA 15-3 were normal.

With this diagnosis, the patient was referred to our medical oncology department where she received postoperative adjuvant chemotherapy consisting of 4 cycles of adriamycin $60 \mathrm{mg} / \mathrm{m}^{2}$ and cyclophosphamide $600 \mathrm{mg} / \mathrm{m}^{2}$ every 3 weeks. Then, adjuvant radiotherapy (50 Gy) was performed on right-breast residual tissue, followed by adjuvant hormone therapy with tamoxifen $20 \mathrm{mg}$ daily during the following 4 years. Meanwhile, the patient continued routine follow-up visits in our department.

In January 2008 (4 years after surgery), the patient noticed the presence of periareolar thickening in the right breast with redness and heat. In the physical examination there was neither a lymph node palpable nor an underlying tumor. A mammography showed the presence of breast architectural distortion with high suspicion of malignancy (BIRADS-4). The result of the biopsy of the lesion confirmed the presence of an IDC consistent with the diagnosis of inflammatory breast cancer $\left(\mathrm{pT}_{4} \mathrm{~N}_{\mathrm{X}}\right.$ $\mathrm{M}_{0}$ ) in the same breast where the previous tumor was treated. Immunohistochemical study of biopsy sample showed negative staining for both ER and PR, and showed positive membrane staining of Her2 marker. FISH analysis found HER-2 gene amplification.

Then, the patient received treatment with a weekly schedule of paclitaxel $\left(80 \mathrm{mg} / \mathrm{m}^{2}\right)$ plus carboplatin (AUC = 2) 3 weeks on and 1 week off, in combination with weekly trastuzumab (initially 4 $\mathrm{mg} / \mathrm{kg}$ followed by $2 \mathrm{mg} / \mathrm{kg}$ every week). She received a total of 11 cycles of chemotherapy schedule and 15 cycles of trastuzumab. During that time period, CA 15-3 serum levels remained normal.

After completing treatment, there was a progressive decrease of inflammatory signs, with disappearance of the redness and heat from the affected area. This allowed the realization of a radical mastectomy in June 2008. The pathology report showed no signs of malignancy with a complete pathological response.

After surgery, the patient received adjuvant chemotherapy consisting of 8 cycles of weekly paclitaxel $\left(80 \mathrm{mg} / \mathrm{m}^{2}\right)$ in combination with weekly trastuzumab $(2 \mathrm{mg} / \mathrm{kg})$, followed by trastuzumab monotherapy ( $6 \mathrm{mg} / \mathrm{kg}$ every 3 weeks) up to 1 year of treatment.

Later, the patient underwent breast reconstruction surgery, and currently, after 4 years and 8 months of follow-up after the last surgery, the patient remains in complete clinical remission, developing her life normally. However, given the age of the patient at the time of first diagnosis and due to the presence of a second primary tumor in the same breast, a genetic study was performed with negative results for currently known breast cancer-associated genetic mutations. 


\section{Discussion}

Breast cancer is a major public health problem for women throughout the world [5], and IDC is the most frequent form of invasive breast cancer. It accounts for $70-80 \%$ of all cases of invasive carcinomas, and, in global, is the histologic type of breast cancer with the worst prognosis of all $[6,7]$. Its treatment is based on a multidisciplinary approach consisting in primary tumor surgery, adjuvant chemotherapy and radiotherapy, and hormone therapy if indicated.

Currently, the treatment of choice for IDC is a combination therapy based on neoadjuvant chemotherapy, targeted therapy, surgery, adjuvant chemotherapy, hormone therapy and radiation therapy.

Numerous randomized prospective studies have shown that breast-conserving treatment in IDC is as effective as mastectomy in terms of overall survival, disease-free survival and long-term disease-free survival $[8,9]$.

Inflammatory breast carcinoma is a rare and very aggressive form of locally advanced breast carcinomas. It represents $0.5-5 \%$ of primary invasive breast tumors, being more common in Caucasian women under 60 years. In these cases, it is important to make a differential diagnosis with locally advanced 'noninflammatory' breast carcinomas that subsequently evolve with inflammation, as well as other nonneoplastic diseases (mastitis and breast abscess), by using biopsies and imaging tests to confirm the differential clinical diagnosis.

After breast-conserving treatment, IBTR may represent two distinct types of lesion that it is important to define, a TR or an NPT. TR and NPT have different natural histories, prognosis, and in turn different implications for therapeutic management [14].

In a retrospective study, designed by Bouchardy et al. [10], about second primary contralateral breast cancer, it is concluded that women with ER-positive first tumors have a decreased risk of second breast cancer occurrence (standardized incidence ratio (SIR): 0.67; 95\% confidence interval (CI), 0.48-0.90), whereas patients with ERnegative primary tumors have an increased risk limited to ER-negative second tumors (SIR: 7.94; 95\% CI, 3.81-14.60). Patients with positive family history had a tenfold higher risk of ER-negative second tumor which increased to nearly 50 -fold when the first tumor was ER negative. So the risk of second ER-negative breast cancer is really very high after a first ER-negative tumor, particularly in women with strong family history [10].

Certain studies provide additional evidence on differences between ER-positive and ER-negative breast cancer, not only in presentation, prognosis, and treatment, but also in etiology and natural history [10].

Overall, the risk of developing a second breast cancer among women diagnosed with a first breast cancer of any ER status was similar to the risk of developing a first breast cancer in the general population. But it is important to analyze the first breast cancer by means of immunohistochemical markers, because risk of a second tumor depends on ER status, period of diagnosis, and family history of breast and/or ovarian cancer; and 
consequently, surveillance and prevention of second cancer occurrence should consider these risk factors.

The risk of a second breast cancer is the same whether the patient had previously underwent a mastectomy or BCT. And with BCT, the risk for developing a second primary breast cancer in the preserved breast is similar to the contralateral one. Unlike disease recurrence, second primary breast cancer often occurs after the first 36 months, with an average range from 34 to 60 months, requiring a long-term follow-up [10].

Secondary malignancies of the breast are rare with a reported frequency of 0.4 to $2.16 \%$. According to the National Surgical Adjuvant Breast and Bowel Project (NSABP), there is a $14.3 \%$ cumulative incidence of IBTR over 20 years since primary operation. IBTR can be defined as the re-emergence of tumor in the previously treated breast. IBTR rate is under $1 \%$ per year, but it is about 5 to $10 \%$ at 5 years and 10 to $15 \%$ at 10 years [11].

When evaluating IBTR, it is important to consider whether it is a TR or an NPT. On the one hand, TR are cases consistent with the regrowth of malignant cells not removed by surgery or not killed by radiotherapy, as Veronesi et al. [8] defined them. On the other hand, NPTs are de novo cases of malignancies arising from mammary epithelial cells of the residual breast tissue $[4,8]$.

The complex behavior of IBTR may be related to the fact that the IBTR patient population is composed by these two different entities.

Each IBTR can be classified as either TR or as NPT based on the following criteria:

\section{Histological Criteria}

According to pathologic features, NPT is designated when it is a distinct histology type (e.g. from an infiltrating ductal carcinoma to an infiltrating lobular, tubular, or medullar carcinoma), or when it has a change from a more invasive to a less invasive carcinoma. By contrast, a change in histology from ductal carcinoma in situ to an infiltrating ductal carcinoma is considered as TR because it is consistent with the natural progression of the disease.

\section{Location Criteria}

Depending on whether the relapse occurs at or near the original site of the primary tumor. It is classified as NPT if it is located in a different quadrant.

\section{DNA Flow Cytometry Criteria}

A tumor is classified as NPT if it changes from an aneuploid primary to a diploid relapse; otherwise, it would be considered as a probable progression representing the natural history.

The best way to differentiate NPT and TR would be genetic sequencing (to establish true clonality). Recently, some molecular techniques such as DNA finger printing, loss of heterozygosity pattern or allelic imbalances profile have been used to distinguish NPT from TR, but the classification criteria are not standardized yet. 
Patients with NPT were significantly younger at initial diagnosis than those who experienced TR. However, there are no age differences at relapse. The rate of NP in the ipsilateral breast does not differ significantly from the rate of primary contralateral tumors.

Currently, salvage mastectomy is the standard treatment for all types of IBTR, which provides locoregional control in $90 \%$ of patients. This recommendation is based on an elevated risk of further in-breast relapse with conservative surgeries. But, there is no conclusive evidence of its superiority compared to conservative surgery, the number of repeat lumpectomies is small and the follow-up is not long enough to draw definitive conclusions. Further studies are needed to determine the indications [12].

After the surgical management of IBTR, the optimal systemic therapy is also unknown.

TR has a poor prognosis in terms of survival rates and development of other metastases, and may benefit from more aggressive adjuvant treatment, with additional radiotherapy, hormone therapy and chemotherapy.

By contrast, patients with NPT generally have a favorable prognosis, and therapeutic decisions concerning systemic therapy should be similar to those in patients with de novo breast carcinoma, according to the equivalent stage. However, because of the higher risk of developing contralateral breast carcinoma (genetic predisposition) there is a need for better chemoprevention strategies. Two trials have shown that patients with NPT benefit from adjuvant tamoxifen, if indicated, because it reduces contralateral and ipsilateral disease recurrence with minimal side effects [13].

Due to declining mortality rates that are, in part, attributable to the use of screening mammography and effective adjuvant therapy, more women are surviving their breast cancer. The care of breast cancer survivors is an important issue that requires an understanding of relapse patterns, establishing appropriate follow-up visits and screening tests. Nowadays, there exists a good follow-up among cancer survivors, with higher screening rates.

\section{References}

1 Yoshida T, Takei H, Kurosumi M, et al: True recurrences and new primary tumors have different clinical features in invasive breast cancer patients with ipsilateral breast tumor relapse after breast-conserving treatment. Breast J 2010;16:127-133.

-2 Klingen TA, Klaasen H, Aas H, et al: Secondary breast cancer: a 5-year population-based study with review of the literature. APMIS 2009;117:762-767.

3 Abd-Alla HM, Lotayef MM, Abou Bakr A, Moneer MM: Ipsilateral in-breast tumor relapse after breast conservation therapy: true recurrence versus new primary tumor. J Egypt Natl Canc Inst 2006;18:183190

-4 Komoike Y, Akiyama F, Iino Y, et al: Analysis of ipsilateral breast tumor recurrences after breastconserving treatment based on the classification of true recurrences and new primary tumors. Breast Cancer 2005; 12:104-111. 
5 Ferlay J, Autier P, Boniol M, et al: Estimates of the cancer incidence and mortality in Europe in 2006. Ann Oncol 2007;18:581-592.

6 Li CI, Uribe DJ, Daling JR: Clinical characteristics of different histologic types of breast cancer. Br J Cancer 2005;93:1046-1052.

7 Elston CW, Ellis IO: Pathological prognostic factors in breast cancer. I. The value of histological grade in breast cancer: experience from a large study with long-term follow-up. C.W. Elston \& I.O. Ellis. Histopathology 1991;19:403-410. Histopathology 2002;41:151-152, discussion 152-153.

$>8$ Veronesi U, Cascinelli N, Mariani L, et al: Twenty-year follow-up of a randomized study comparing breast-conserving surgery with radical mastectomy for early breast cancer. $\mathrm{N}$ Engl J Med 2002;347:1227-1232

$\$ 9$ Fisher B, Anderson S, Bryant J, et al: Twenty-year follow-up of a randomized trial comparing total mastectomy, lumpectomy, and lumpectomy plus irradiation for the treatment of invasive breast cancer. N Engl J Med 2002;347:1233-1241.

-10 Bouchardy C, Benhamou S, Fioretta G, et al: Risk of second breast cancer according to estrogen receptor status and family history. Breast Cancer Res Treat 2011;127:233-241.

11 Fowble B: Ipsilateral breast tumor recurrence following breast-conserving surgery for early-stage invasive cancer. Acta Oncol 1999;38(suppl 13):9-17.

12 Salvadori B, Marubini E, Miceli R, et al: Reoperation for locally recurrent breast cancer in patients previously treated with conservative surgery. Br J Surg 1999;86:84-87.

13 Dalberg K, Johansson H, Johansson U, Rutqvist LE: A randomized trial of long term adjuvant tamoxifen plus postoperative radiation therapy versus radiation therapy alone for patients with early stage breast carcinoma treated with breast-conserving surgery. Stockholm Breast Cancer Study Group. Cancer 1998;82:2204-2211. 